Supporting Information

\title{
Stimulus-Responsive Graphene with Periodical Wrinkles on Grooved Microfibers Arrays: Simulation, Programmable Shape-Shifting, and Catalytic Applications
}

Xiangyu Meng,a, c Jianhui Yang, ${ }^{a}$ Wei Liu, ${ }^{\mathrm{b}}$ Seeram Ramakrishna, ${ }^{\mathrm{d}}$ Yueming Sun, ${ }^{\mathrm{a}}$ and Yunqian Dai*,a, c

aSchool of Chemistry and Chemical Engineering, Southeast University, Nanjing, Jiangsu 211189, P. R. China

${ }^{b}$ School of Instrument Science and Engineering, Southeast University, Nanjing, Jiangsu 211189, P. R. China

${ }^{\mathrm{c}}$ Center for Flexible RF Technology, Southeast University, Purple Mountain Laboratory, Nanjing, Jiangsu, 211189, People's Republic of China

${ }^{\mathrm{d} C e n t e r}$ for Nanotechnology \& Sustainability, Department of Mechanical Engineering, National University of Singapore, 119260, Singapore

*Address corresponding to: daiy@seu.edu.cn 
Note S1. Calculation of alignment degree of CA mat

The alignment degree $(d)$ of our mat was calculated from SEM image in Figure 1D and based on equation $\mathrm{S} 1: \mathrm{S} 1, \mathrm{~S} 2$

$$
d=\frac{3 \cos ^{2} \theta-1}{2}
$$

where $\theta$ is the average angle between individual CA microfiber and preferred alignment direction of the electrospun mat. The value of $\theta$ was measured as $6.67^{\circ}$. The value of $d$ was calculated as 0.97 . 


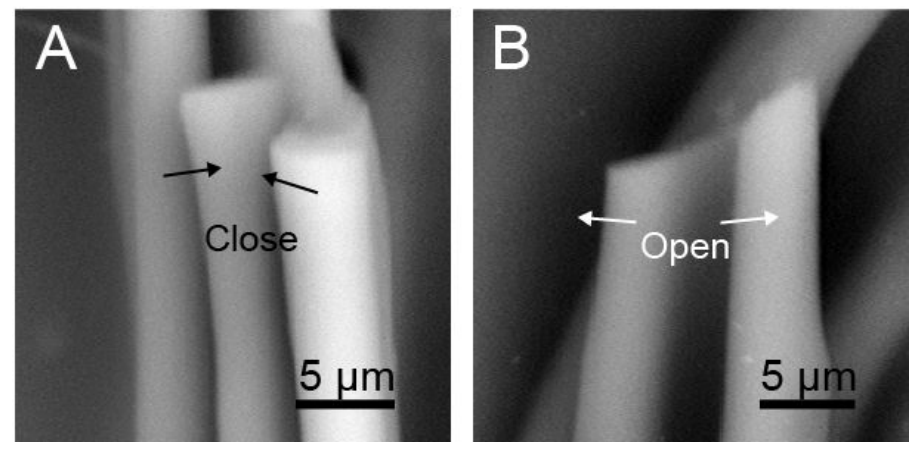

Figure S1. (A) SEM images of grooved CA microfibers before and (B) after swelling, showing the in situ opening process of grooves under humidity-stimuli. 


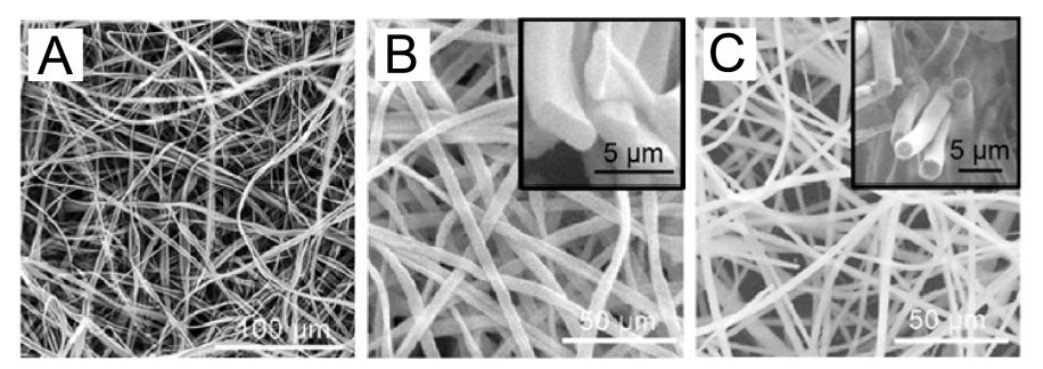

Figure S2. (A) SEM image of the nonwoven grooved CA fibrous mat fabricated without rotating drum. (B) SEM image of the CA microfibers with ribbon-like shape and (C) cylindrical shape. 

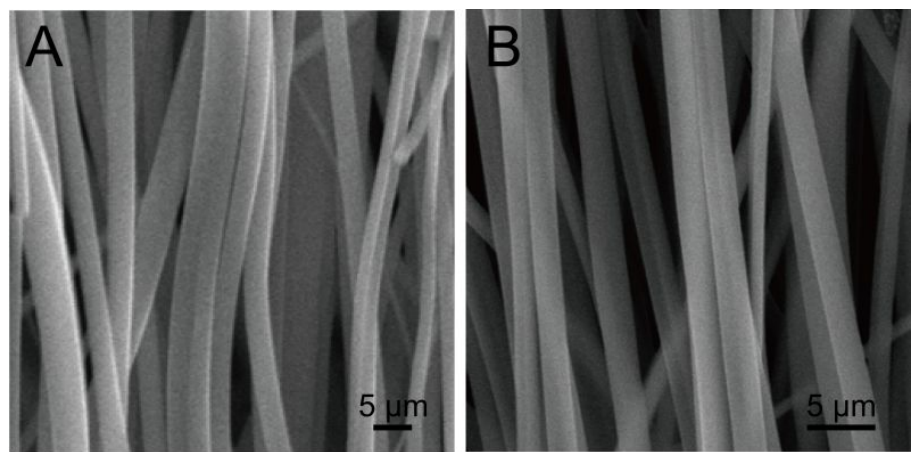

Figure S3. (A) SEM image of the aligned CA fibrous mat with ribbon-like shape and (B) cylindrical shape. 


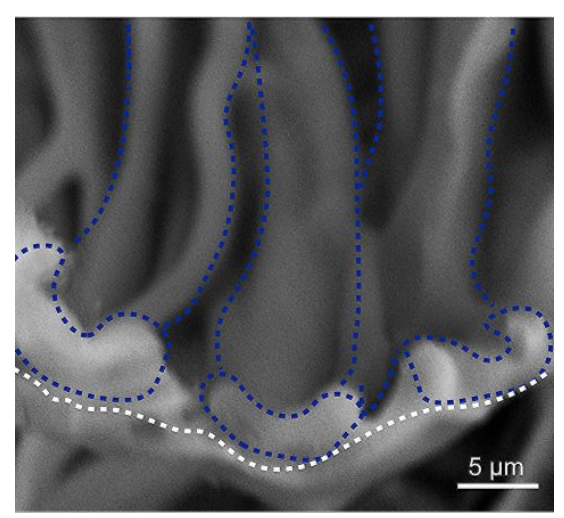

Figure S4. SEM image from the cross-section view, showing the aligned CA microfibers (highlighted by blue lines) directly deposited on a collecting conductive tape (highlighted by the white line) adhered on the rotating drum. 


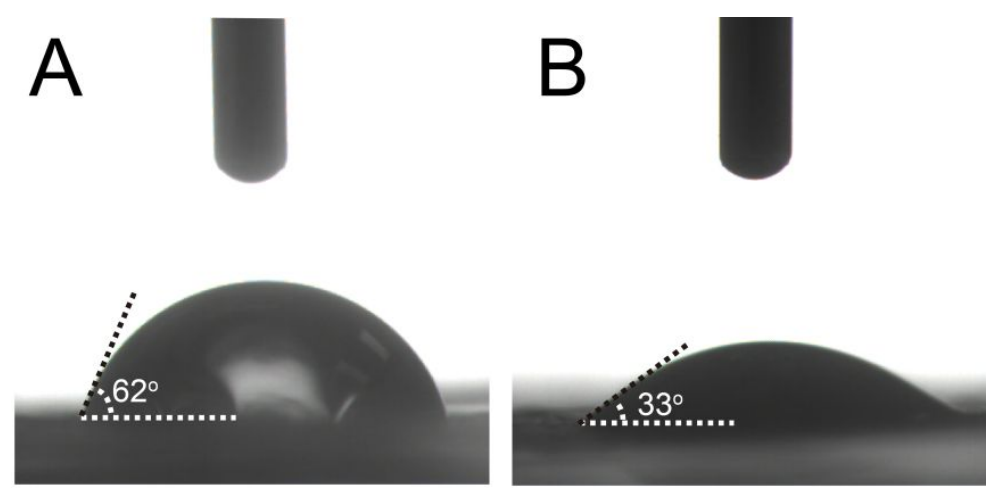

Figure S5. (A) Water contact angle of random and (B) aligned CA fibrous mat. 


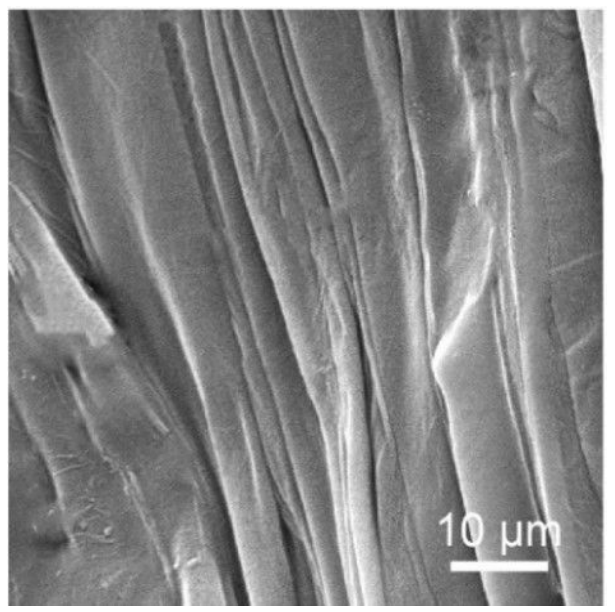

Figure S6. SEM image of wet GO/CA mat, showing the enlarged contact area between GO and CA after swelling. 

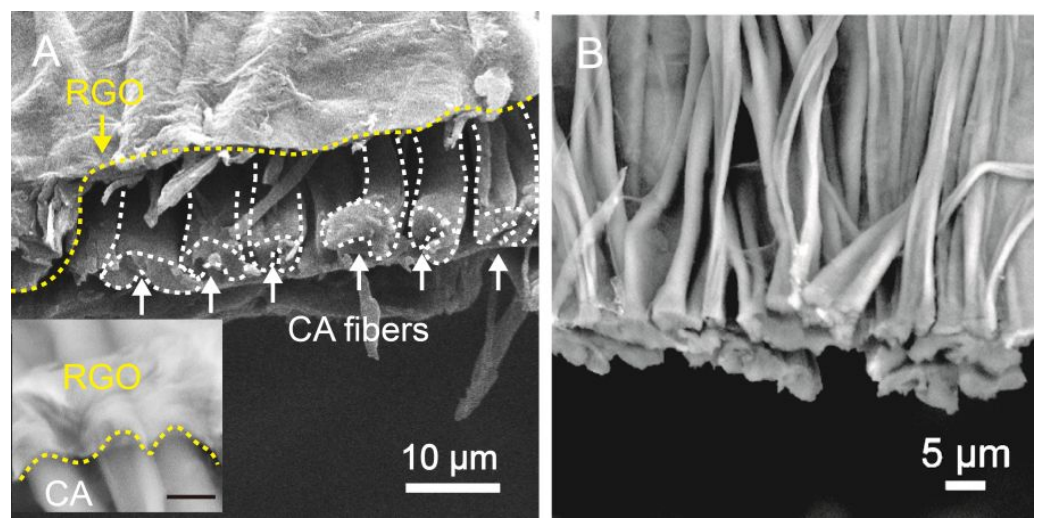

Figure S7. (A) SEM image of RGO/CA after hot-pressing and partially peeling of RGO, showing the well-maintained aligned grooved fibers. The inset shows the conformal structure of RGO/CA after hot-pressing and partially peeling of RGO, with a scale bar of $10 \mu \mathrm{m}$. The yellow and white dashed lines highlight the RGO layer after peeling-off and the CA fibers, respectively. (B) SEM image of aligned grooved CA microfibers after hot-pressing. 


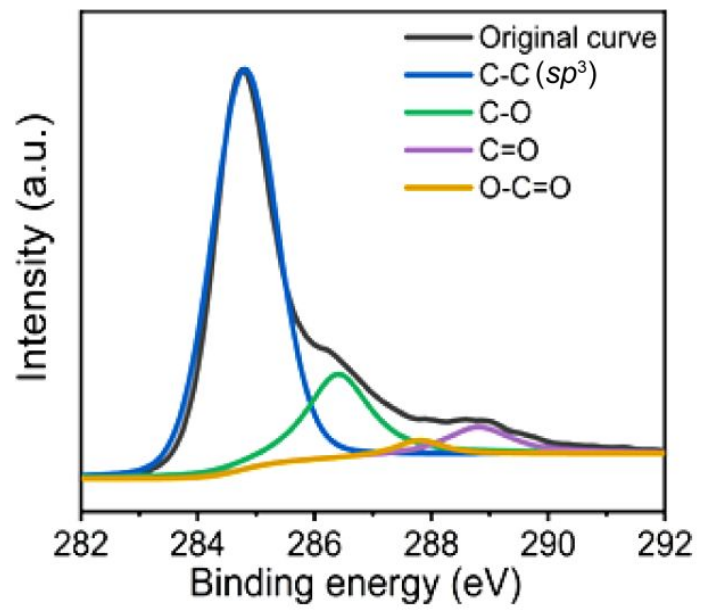

Figure S8. C $1 s$ high-resolution XPS spectrum of RGO. 


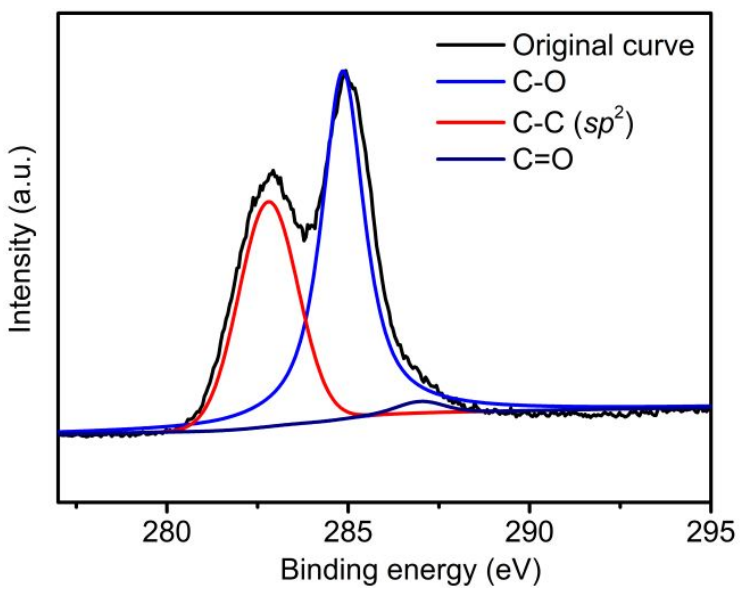

Figure S9. C $1 s$ high-resolution XPS spectrum of GO. 


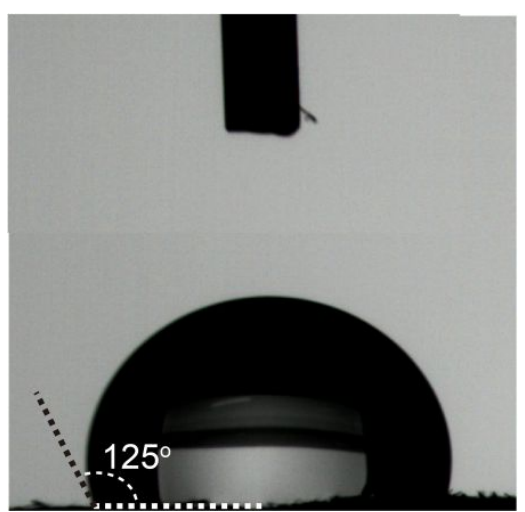

Figure S10. Water contact angle of RGO. 


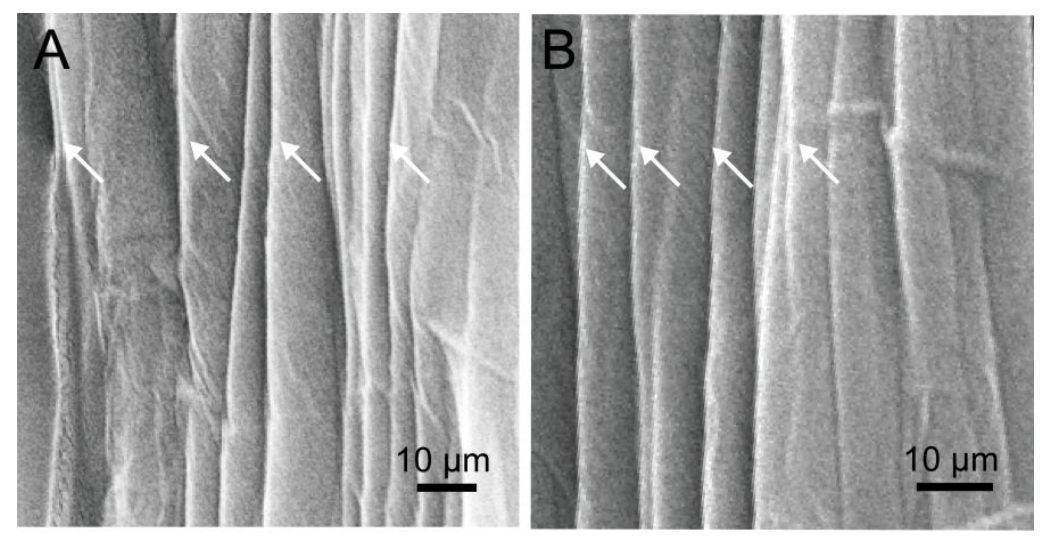

Figure S11. (A) SEM image of RGO/CA mat with wrinkled structures on RGO before and (B) after the 20-times bending deformations with a large angle $\left(120^{\circ}\right)$. The white arrows highlight the wrinkles. 

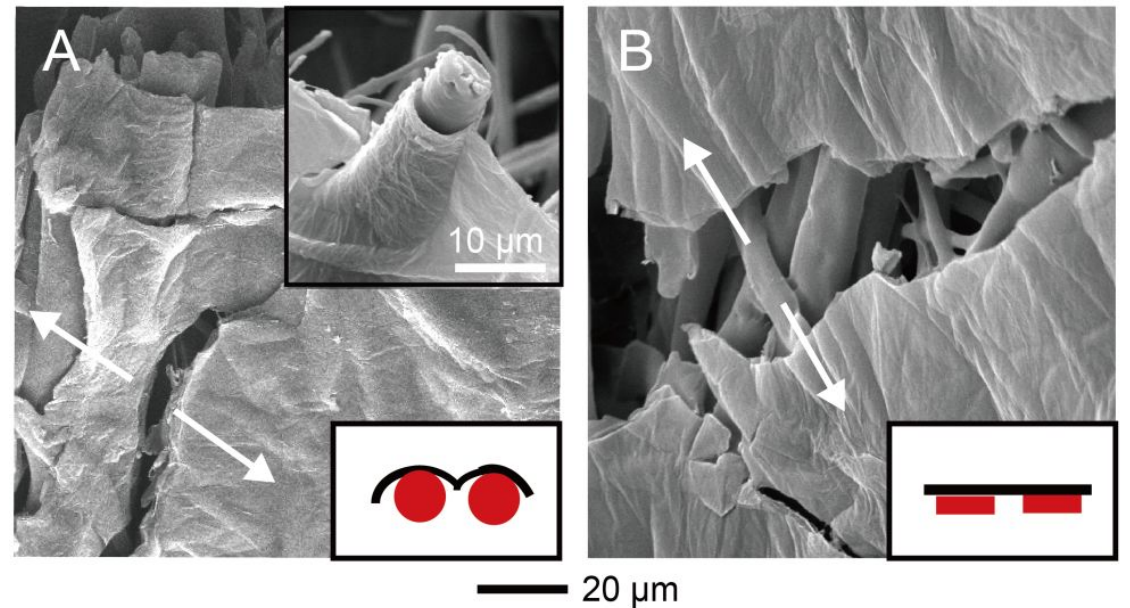

Figure S12. (A) SEM images of RGO/cylindrical CA mat and (B) RGO/ribbon-like CA mat after 20 -times bending deformations with a large angle $\left(120^{\circ}\right)$. The white arrows highlight the interfacial slipping of bi-layers. The insets illustrate the weak combination without interlocking behavior of grooved CA microfibers. 
Table S1. Comparison of the mechanical properties between this work and previous works of soft materials.

\begin{tabular}{|c|c|c|c|c|}
\hline Materials & $\begin{array}{l}\text { Young's } \\
\text { (MPa) }\end{array}$ & modulus & $\begin{array}{r}\text { Breaking } \\
\text { stress (MPa) }\end{array}$ & Reference \\
\hline $\mathrm{RGO} / \mathrm{CA}$ & 820 & & 18 & This work \\
\hline Polyampholyte hydrogel & $<0.1$ & & 0.25 & $\mathrm{~S} 3$ \\
\hline Liquid Crystal Fibers & 2 & & 4 & S4 \\
\hline Liquid Crystal Actuator & 3.1 & & 0.3 & S5 \\
\hline $\mathrm{OEG} / \mathrm{cPEGDA} / \mathrm{W}_{18} \mathrm{O}_{49}$ & 4.5 & & 12 & S6 \\
\hline Ionic composite hydrogel & $<0.01$ & & 7 & S7 \\
\hline
\end{tabular}

OEG represents oligo(ethylene glycol), cPEGDA represents the cross-linked polyethylene glycol diacrylate. Data were given or calculated in the respective references. 


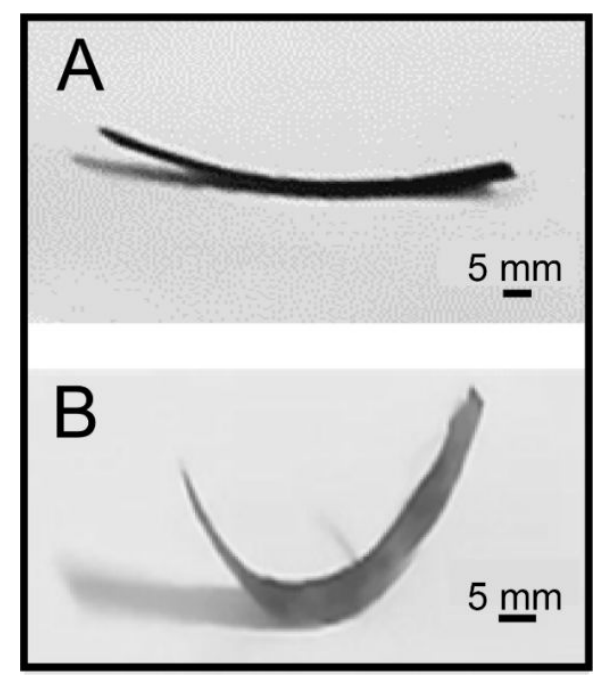

Figure S13. (A) The digital photo of the response to the humidity of RGO/CA/RGO mat and (B) RGO/CA mat. 
A
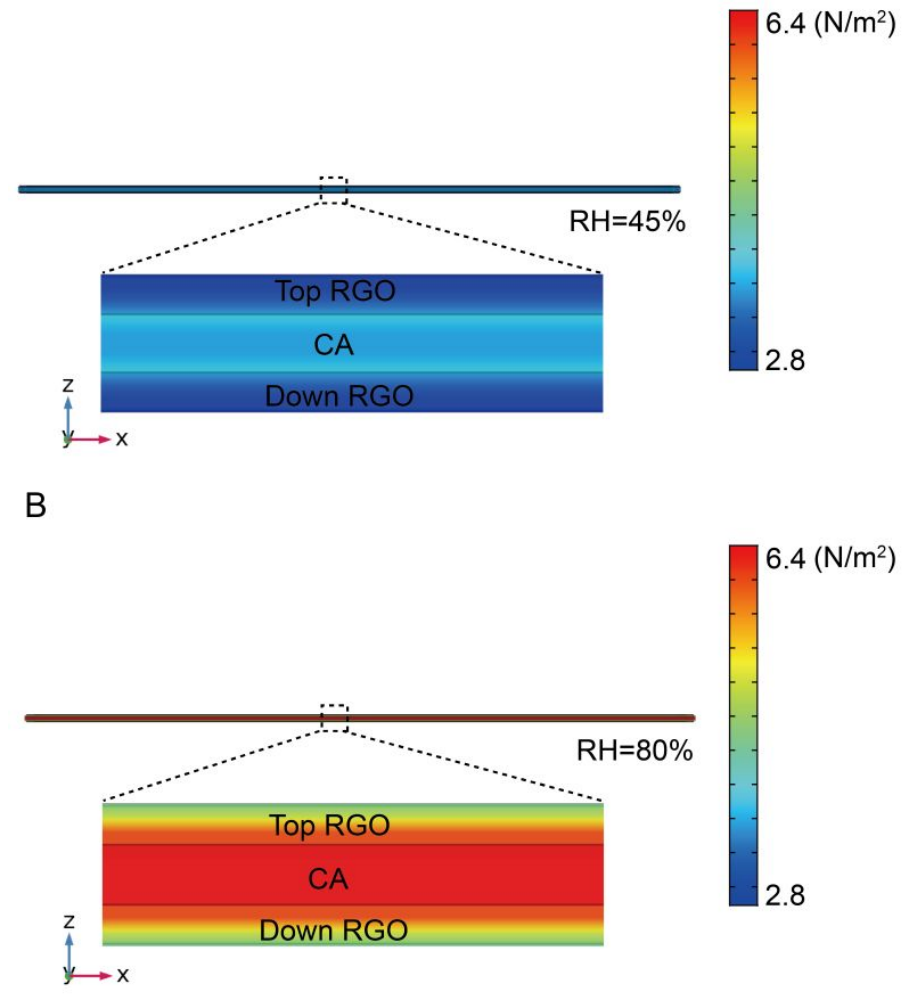

Figure S14. (A) Simulated deformation and stress distribution of RGO/CA/RGO upon receiving humidity stimulus under relative humidity of $45 \%$ and (B) $80 \%$. The color gradients represent the stress $\left(\mathrm{N} / \mathrm{m}^{2}\right)$, the states of models represent the real deformation states in simulations. 
Note S2. The coefficient of hygroscopic expansion (CHE) were calculated by equation S2. CHE

$$
=\frac{\Delta L}{\Delta H \times L}=\frac{\Delta \mathrm{R}}{\mathrm{R} \times \Delta H}
$$

where $L(\mathrm{~m})$ is the length of CA mat, $R(\mathrm{~m})$ is the curvature radius of $\mathrm{RGO} / \mathrm{CA}$ mat, $H(\%)$ is the relative humidity. 
Table S2. Comparison of the coefficient of hygroscopic expansions (CHE) between this work and reported works.

\begin{tabular}{lll}
\hline Materials & CHE & Reference \\
\hline Aligned RGO/CA mat & $3.06 \times 10^{-3}$ & This work \\
Random RGO/CA mat & $1.80 \times 10^{-3}$ & This work \\
PANI-S/PI & $0.13 \times 10^{-3}$ & $\mathrm{~S} 8$ \\
PEDOT: PSS & $0.47 \times 10^{-3}$ & $\mathrm{~S} 9$ \\
PPy/AG & $1 \times 10^{-3}$ & $\mathrm{~S} 10$ \\
BOPP/Paper & $0.23 \times 10^{-3}$ & $\mathrm{~S} 11$ \\
SGO/PVDF & $0.20 \times 10^{-3}$ & $\mathrm{~S} 12$ \\
\hline
\end{tabular}

Data were given or calculated in the respective references. 


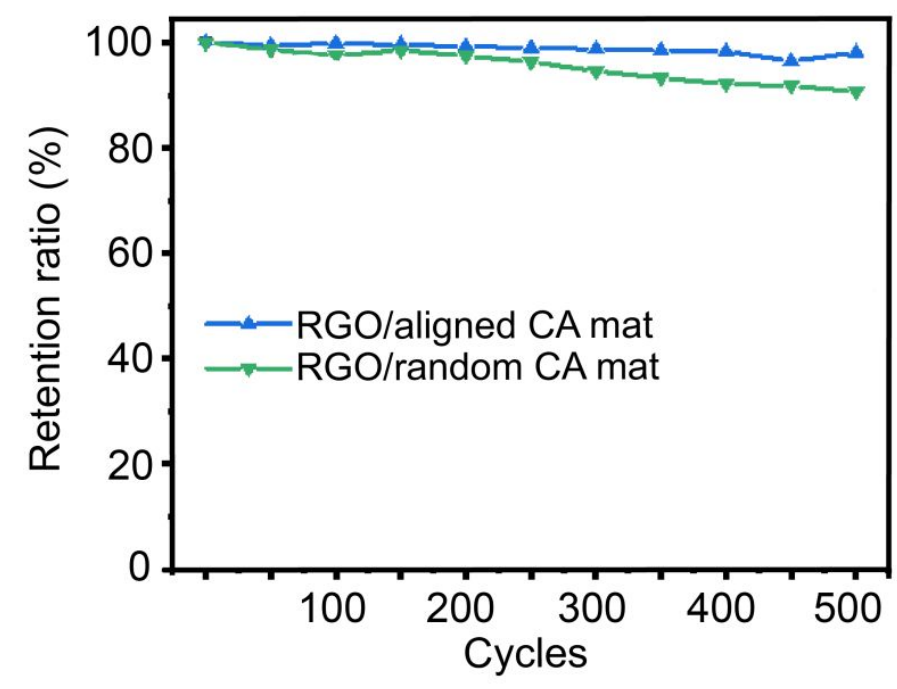

Figure S15. The cycling test curves under humidity-stimuli. 
Note S3. The friction analysis during the humidity-induced deformation of aligned and random $\mathrm{RGO} / \mathrm{CA}$ mat.

Since the humidity-responsive actuation was induced by the anisotropic swelling of CA microfibers, the friction analysis of RGO/CA was simplified and based on the deformation of CA microfibers. Assuming the swelling elongation of each CA microfiber along its long-axis is A\% and the average angle between random CA microfibers was $\theta$, the deformation of CA microfiber along the $\mathrm{x}$ - and $\mathrm{y}$-axis are shown in the following Scheme S1:

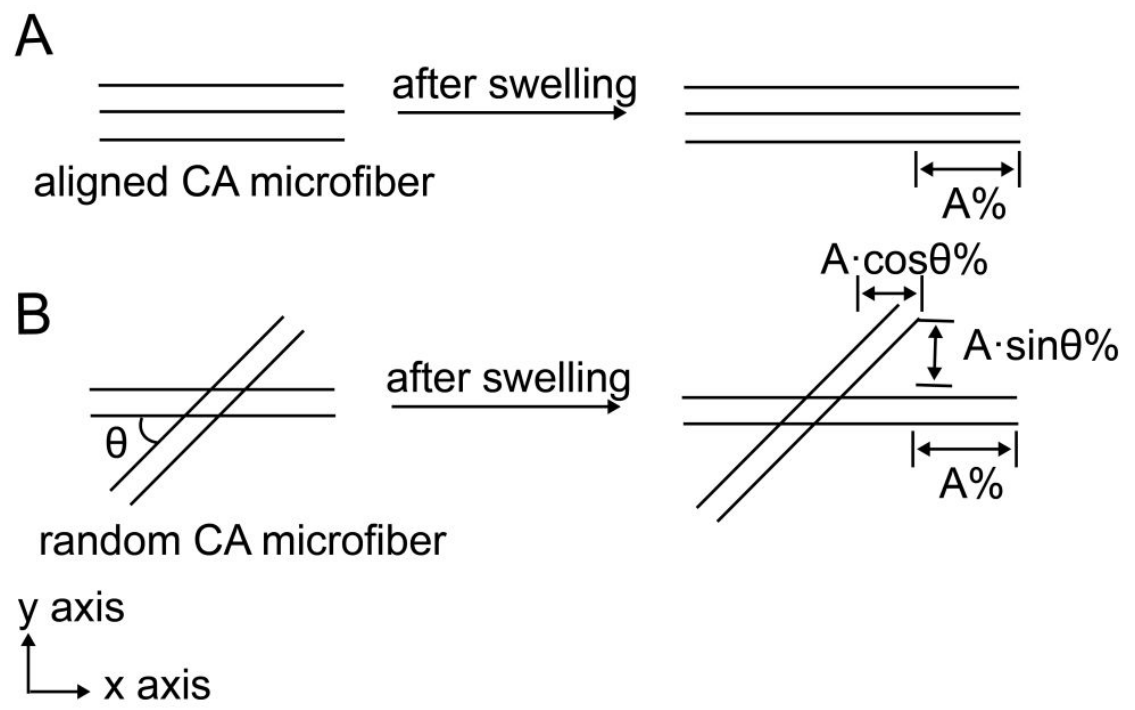

Scheme S1. (A) The schematic illustration of swelling deformation of aligned and (B) random CA microfibers.

The swelling deformation of aligned CA microfibers is synchronous on both $\mathrm{x}$ (i.e., $|\mathrm{A}| \%$ ) and y axis (i.e., $0 \%$, which causes nearly no relative motions and frictions. In the case of random CA microfibers, one fiber elongates $|\mathrm{A}| \%$ along the $\mathrm{x}$ axis and $0 \%$ along the $\mathrm{y}$ axis. The other fiber elongates $|A \cdot \cos \theta| \%$ along the $\mathrm{x}$ axis and $|\mathrm{A} \cdot \sin \theta| \%$ along the $\mathrm{y}$ axis. The relative motions could cause frictions on the interface between $\mathrm{RGO} / \mathrm{CA}$ random microfibers. Hence, the aligned CAbased mats could suffer from decreased internal energy upon less friction compared to random CA-based mats. 

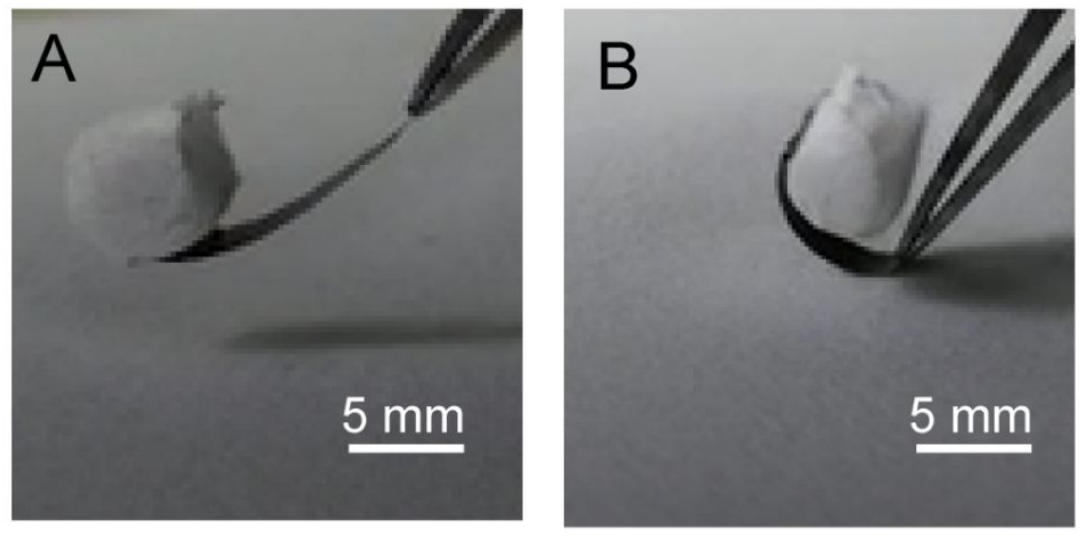

C

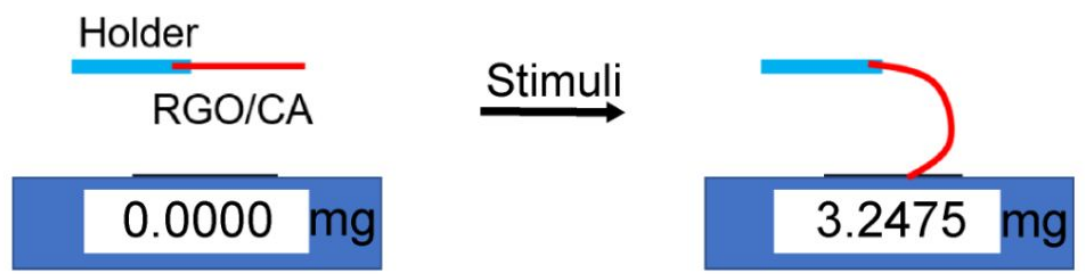

Figure S16. (A, B) Digital photos of the lifting process under humidity stimulus. (C) The schematic image of the measuring of output force, which was tested in a relative humidity of $80 \%$ by the microbalance. 

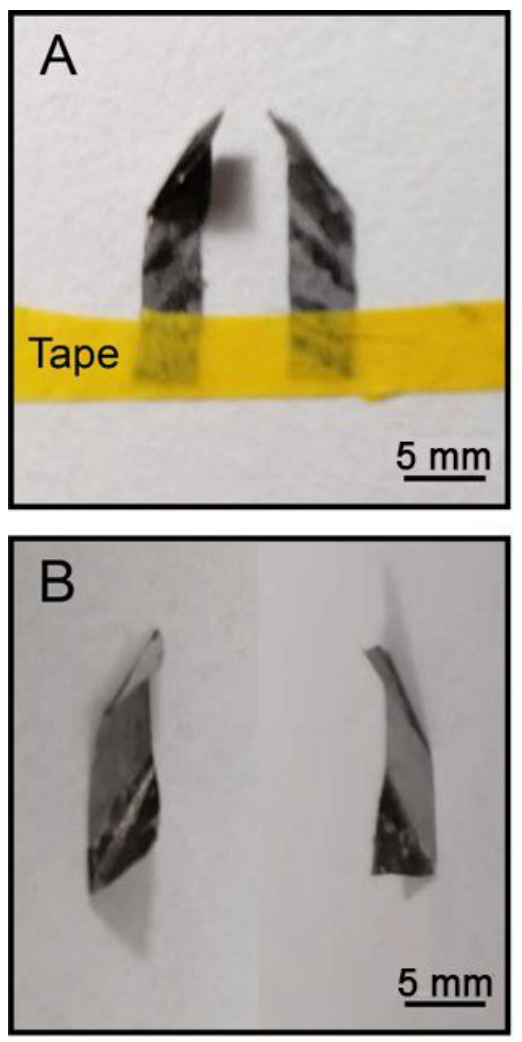

Figure S17. (A) The chirality of programmed deformations achieved by the RGO/CA with barshaped RGO patterns and (B) with triangle-shaped RGO patterns. 


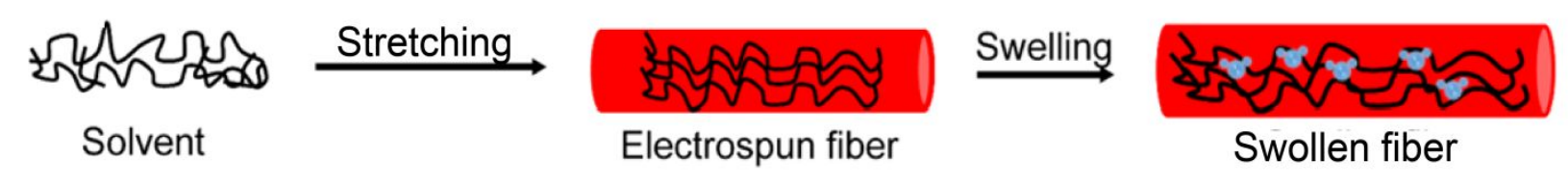

Figure S18. Schematic illustration of the mechanism of anisotropic swelling behavior in each CA microfiber. 
Table S3. Comparison of the photothermal conversion efficiency between this work and previous works of light-responsive materials.

\begin{tabular}{lll}
\hline Materials & Photothermal conversion efficiency & Reference \\
\hline RGO & $73.5 \%$ & This work \\
Cu nano rods & $27.9 \%$ & $\mathrm{~S} 13$ \\
$\mathrm{MoS}_{2}$ & $47.0 \%$ & $\mathrm{~S} 14$ \\
$\mathrm{CNT} /$ Paraffin wax/Polyimide & $62.5 \%$ & $\mathrm{~S} 15$ \\
$\mathrm{AG} / \mathrm{CNT}$ & $69.0 \%$ & $\mathrm{~S} 16$
\end{tabular}

AG represents agarose, CNT represents the carbon nanotube. Data were given or calculated in the respective references. 


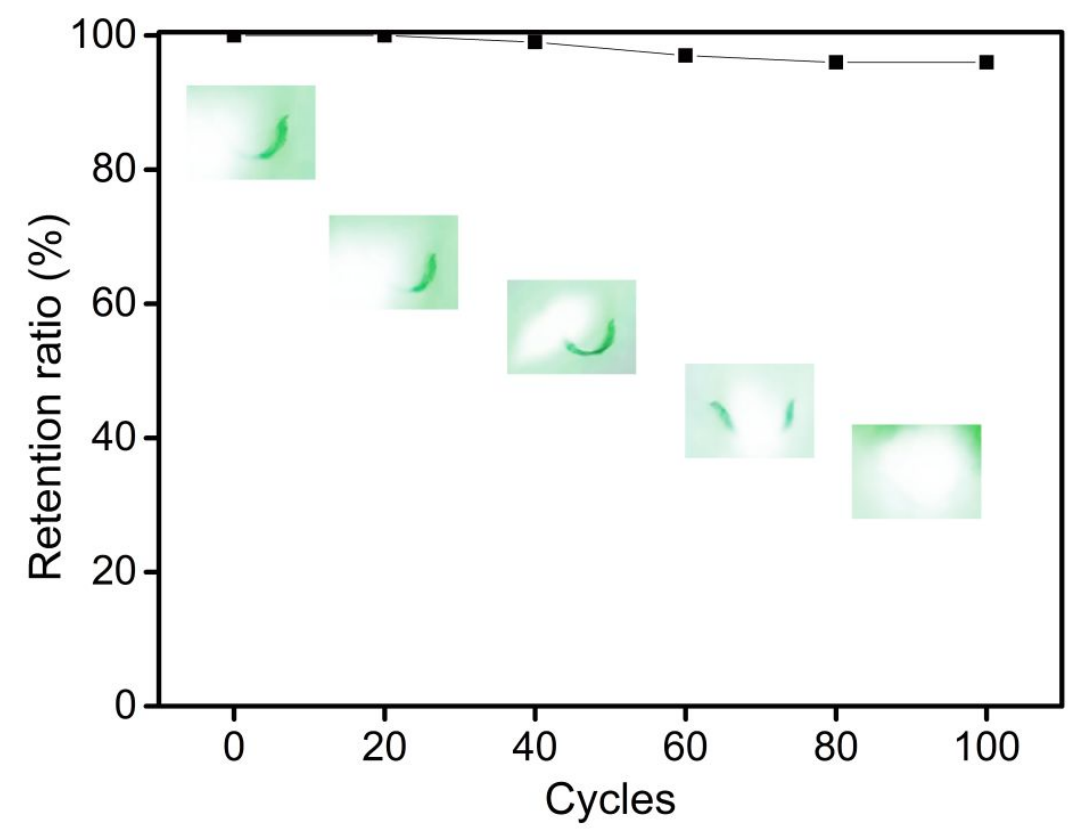

Figure S19. The cycle test of RGO/aligned CA under 100-times repeated light-induced actuations. 


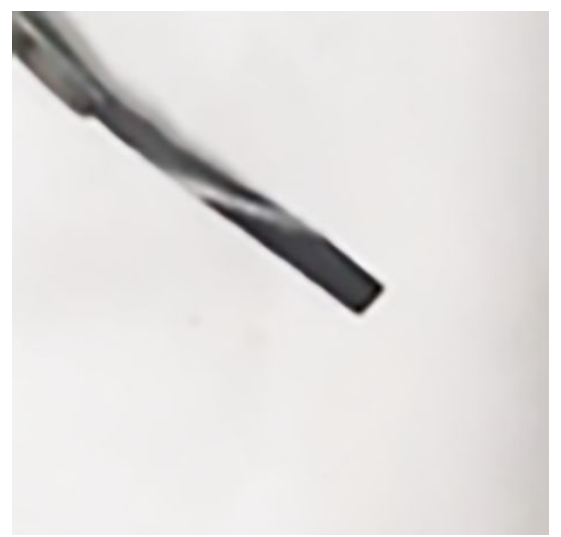

Figure S20. The optical image of smart mat after the deformation and removing both the light and humidity. 

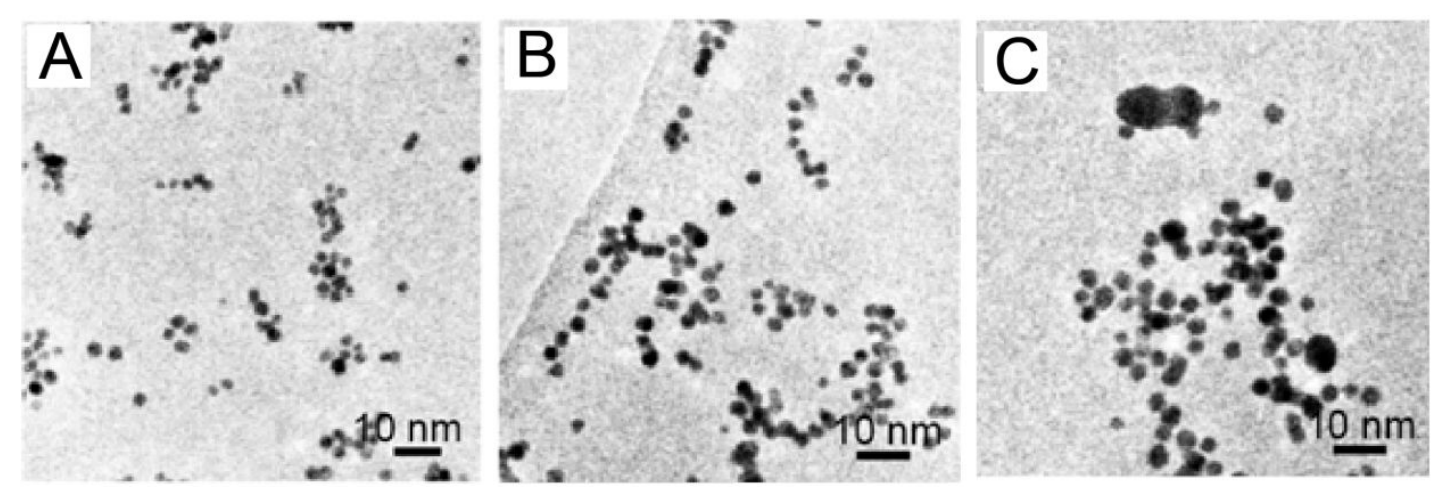

Figure S21. (A) TEM image of the Au loading on CA microfiber. (B) TEM image of the Au nanoparticles which were supported on CA microfibers after catalyzing. (C) TEM image of the Au nanoparticles which were directly released on reaction position after catalyzing, without the protection of the built-in microchannels. 


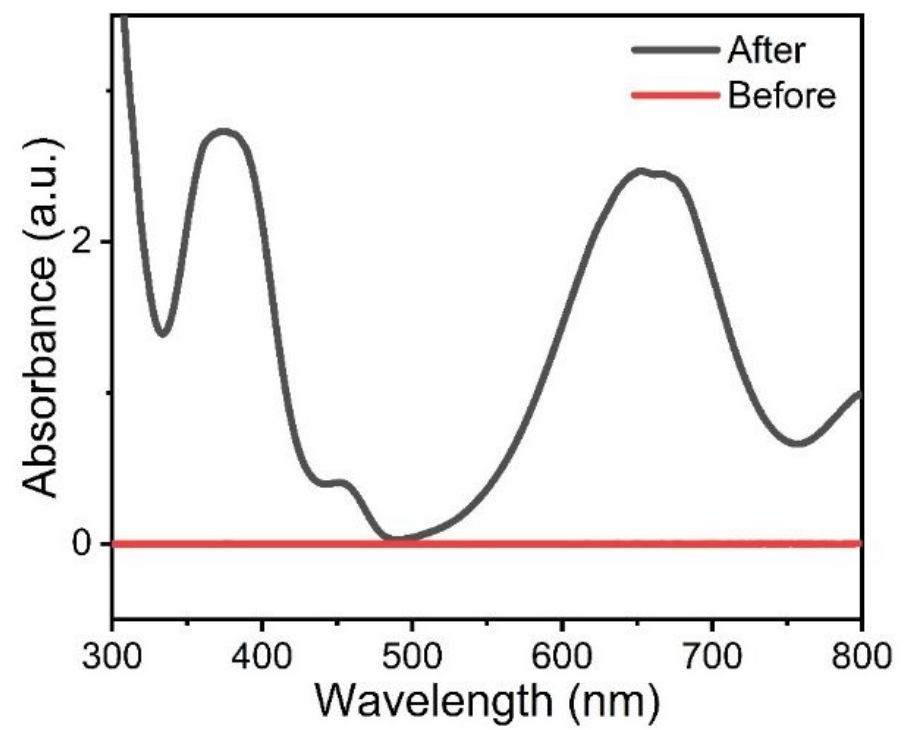

Figure S22. The UV-vis spectrum of reaction solution before and after catalyzing by $\mathrm{Au} / \mathrm{RGO} / \mathrm{CA}$ mat. 


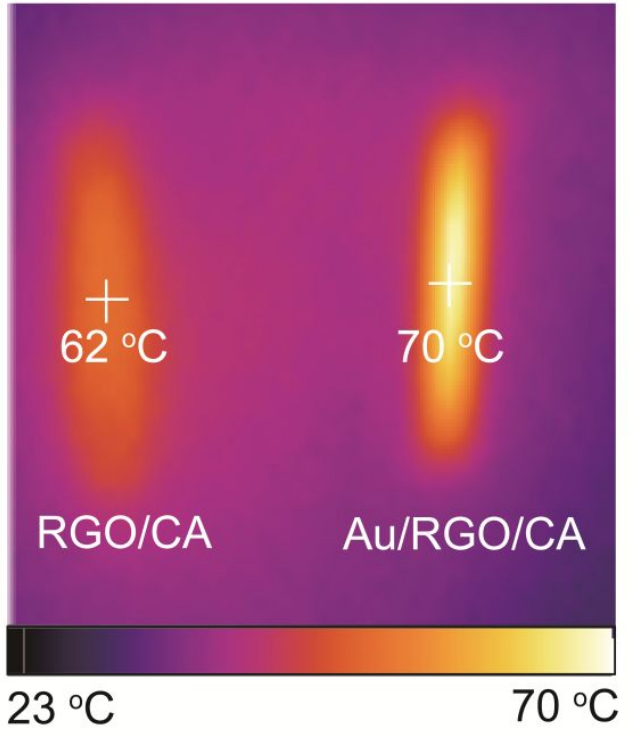

Figure S23. Infrared image of $\mathrm{RGO} / \mathrm{CA}$ and $\mathrm{RGO} / \mathrm{CA}$ loaded with $\mathrm{Au}$ nanoparticles under light irradiation. 


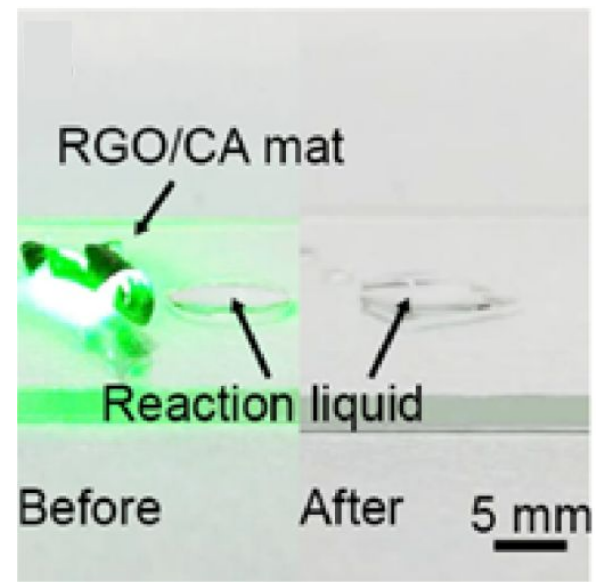

Figure 24. Optical photos of reaction solution before and after contacting with RGO/CA mat. 


\section{Explanatory Notes of Movies:}

Movie S1. The simulated and humidity-induced deformation of RGO/CA. The video is at its 0.2 times speed.

Movie S2. The simulated and light-induced deformation of RGO/CA. The video is at its 0.3 times speed.

Movie S3. The snatch of catalysts to desired position completed by RGO/CA mat, wirelessly driven by remote light. The video is at its three times speed.

Movie S4. The smart catalyzing process completed by RGO/CA mat, wirelessly driven by remote light. The video is at its three times speed. 


\section{References}

(S1) Dersch, R.; Liu, T.; Schaper, A. K.; Greiner, A.; Wendorff, J. H. Electrospun Nanofibers: Internal Structure and Intrinsic Orientation. J. Polym. Sci. A Polym. Chem. 2003, 41, 545-553.

(S2) Liu, L.; Bakhshi, H.; Jiang, S.; Schmalz, H.; Agarwal, S. Composite Polymeric Membranes with Directionally Embedded Fibers for Controlled Dual Actuation. Macromol. Rapid Commun. 2018, 39, 1800082.

(S3) Zhang, Y.; Liao, J.; Wang, T.; Sun, W.; Tong, Z. Polyampholyte Hydrogels with pH Modulated Shape Memory and Spontaneous Actuation. Adv. Funct. Mater. 2018, 28, 1707245.

(S4) Roach, D. J.; Yuan, C.; Kuang, X.; Li, V. C. F.; Blake, P.; Romero, M. L.; Hammel, I.; Yu, K.; Qi, H. J. Long Liquid Crystal Elastomer Fibers with Large Reversible Actuation Strains for Smart Textiles and Artificial Muscles. ACS Appl. Mater. Interfaces 2019, 11, $19514-19521$.

(S5) Kotikian, A.; Truby, R. L.; Boley, J. W.; White, T. J.; Lewis, J. A. 3D Printing of Liquid Crystal Elastomeric Actuators with Spatially Programed Nematic Order. Adv. Mater. 2018, $30,1706164$.

(S6) Zhou, Y.; Tan, J.; Chong, D.; Wan, X.; Zhang, J. Rapid Near-Infrared Light Responsive Shape Memory Polymer Hybrids and Novel Chiral Actuators Based on Photothermal $\mathrm{W}_{18} \mathrm{O}_{49}$ Nanowires. Adv. Funct. Mater. 2019, 29, 1901202.

(S7) Odent, J.; Wallin, T. J.; Pan, W.; Kruemplestaedter, K.; Shepherd, R. F.; Giannelis, E. P. Highly Elastic, Transparent, and Conductive 3D-Printed Ionic Composite Hydrogels. Adv. Funct. Mater. 2017, 27, 1701807.

(S8) Tseng, I. H.; Li, J. J.; Chang, P. Y. Mimosa Pudica Leaf-Like Rapid Movement and Actuation of Organosoluble Polyimide Blending with Sulfonated Polyaniline. Adv. Mater. Interfaces 2017, 4, 1600901. 
(S9) Amjadi, M.; Sitti, M. High-Performance Multiresponsive Paper Actuators. ACS Nano 2016, $10,10202-10210$.

(S10)Li, Y.; Yang, J.; Yu, X.; Sun, X.; Chen, F.; Tang, Z.; Zhu, L.; Qin, G.; Chen, Q. Controlled Shape Deformation of Bilayer Films with Tough Adhesion Between Nanocomposite Hydrogels and Polymer Substrates. J. Mater. Chem. B 2018, 6, 6629-6636.

(S11)Ji, M.; Jiang, N.; Chang, J.; Sun, J. Near-Infrared Light-Driven, Highly Efficient Bilayer Actuators Based on Polydopamine-Modified Reduced Graphene Oxide. Adv. Funct. Mater. 2014, 24, 5412-5419.

(S12)Xu, G.; Zhang, M.; Zhou, Q.; Chen, H.; Gao, T.; Li, C.; Shi, G. A Small Graphene Oxide Sheet/Polyvinylidene Fluoride Bilayer Actuator with Large and Rapid Responses to Multiple Stimuli. Nanoscale 2017, 9, 17465-17470.

(S13) Chen, J.; Feng, J.; Yang, F.; Aleisa, R.; Zhang, Q.; Yin, Y. Space-Confined Seeded Growth of $\mathrm{Cu}$ Nanorods with Strong Surface Plasmon Resonance for Photothermal Actuation. Angew. Chem. Int. Ed. 2019, 58, 9275-9281.

(S14) Lei, Z.; Zhu, W.; Sun, S.; Wu, P. $\mathrm{MoS}_{2}$-Based Dual-Responsive Flexible Anisotropic Actuators. Nanoscale 2016, 8, 18800-18807.

(S15) Deng, J.; Li, J.; Chen, P.; Fang, X.; Sun, X.; Jiang, Y.; Weng, W.; Wang, B.; Peng, H. Tunable Photothermal Actuators Based on a Pre-programmed Aligned Nanostructure. J. Am. Chem. Soc. 2016, 138, 225-230.

(S16) Tu, Y.; Yuan, J.; Lei, D.; Tan, H.; Wei, J.; Huang, W.; Zhang, L. TiO ${ }_{2}$-Pattern-Modulated Actuation of an Agarose@CNT/Agarose Bilayer Induced by Light and Humidity. J. Mater. Chem. A 2018, 6, 8238-8243. 